\title{
An efficient method to make human monoclonal antibodies from memory B cells: potent neutralization of SARS coronavirus
}

\author{
Elisabetta Traggiai ${ }^{1,6}$, Stephan Becker ${ }^{2,6}$, Kanta Subbarao ${ }^{3,6}$, Larissa Kolesnikova ${ }^{2}$, Yasushi Uematsu ${ }^{4}$, \\ Maria Rita Gismondo ${ }^{5}$, Brian R Murphy ${ }^{3}$, Rino Rappuoli ${ }^{4}$ \& Antonio Lanzavecchia ${ }^{1}$
}

\begin{abstract}
Passive serotherapy can confer immediate protection against microbial infection, but methods to rapidly generate human neutralizing monoclonal antibodies are not yet available. We have developed an improved method for Epstein-Barr virus transformation of human B cells. We used this method to analyze the memory repertoire of a patient who recovered from severe acute respiratory syndrome coronavirus (SARS-CoV) infection and to isolate monoclonal antibodies specific for different viral proteins, including 35 antibodies with in vitro neutralizing activity ranging from $10^{-8} \mathrm{M}$ to $10^{-11} \mathrm{M}$. One such antibody confers protection in vivo in a mouse model of SARS$\mathrm{CoV}$ infection. These results show that it is possible to interrogate the memory repertoire of immune donors to rapidly and efficiently isolate neutralizing antibodies that have been selected in the course of natural infection.
\end{abstract}

The emergence and spread of new pathogens and the risk of bioterrorism represent continuous threats for human health. Vaccination can confer active and sustained protection, but the development of new vaccines is a slow process and vaccines are effective only in a preventive setting. The practice of administering polyclonal immunoglobulins from hyperimmune sera of animal or human origin, introduced by Behring and Kitasato ${ }^{1}$, has been used extensively in prophylactic as well as therapeutic settings ${ }^{2}$. However, there are several problems associated with the use of polyclonal sera, such as the antigenicity of heterologous proteins, the difficulty of finding immune donors, and risks related to the use of human blood products.

Monoclonal antibodies represent an ideal alternative to hyperimmune sera ${ }^{3}$. Early work showed that human monoclonal antibodies can be produced by immortalizing B cells with Epstein-Barr virus $(\mathrm{EBV})^{4-6}$ or by fusing B cells with an appropriate partner to produce hybridomas ${ }^{7,8}$. However, these methods have very low efficiency and therefore alternative strategies have been developed. These include (i) humanization of murine monoclonal antibodies through protein engineering ${ }^{9}$, (ii) selection of antibodies from phage-display libraries of human antibody fragments ${ }^{10}$ and (iii) immunization of transgenic mice carrying human immunoglobulin loci, followed by production of monoclonal antibodies using hybridoma technology ${ }^{11}$. Although these methods have led to the development of several therapeutic monoclonal antibodies against cytokines or surface molecules, their impact on infectious disease therapy has been less pronounced ${ }^{12}$. Indeed, the number of therapeutic antibodies against infectious agents is still limited and only one is currently in use, to prevent respiratory syncytial virus (RSV) infection in newborns ${ }^{13}$.

The outbreak of SARS in March 2003 caused by SARS-CoV represents the most recent threat to human health ${ }^{14-17}$. The virus is highly contagious and the clinical course is severe, with a high mortality rate ${ }^{18-20}$. Although the production of a vaccine remains a long-term goal, the benefits of vaccination can be expected only if a large proportion of the population is immunized. However, the low incidence of new cases of SARS suggests that large-scale vaccination may not be the best means of controlling disease spread.

During a SARS outbreak, passive serotherapy can potentially provide immediate protection against infection for contacts and medical personnel; a cure of infected individuals is also possible. Because human sera containing antibodies to SARS-CoV are not available in sufficient amounts, we considered the production of human monoclonal antibodies. Using an improved method of EBV transformation, we immortalized memory B cells from a patient who had recovered from SARS-CoV infection and isolated several monoclonal antibodies with high viral neutralizing activity in vitro and in vivo.

\section{RESULTS}

Serological and cellular response to SARS-CoV

The antibody response of a patient who recovered from SARS-CoV infection was analyzed using three assays: (i) ELISA using SDSextracted viral proteins, (ii) staining of baby hamster kidney (BHK) cells transfected with SARS-CoV spike mRNA and (iii) neutralization

${ }^{1}$ Institute for Research in Biomedicine, Via Vela 6, CH 6500 Belllinzona, Switzerland. ${ }^{2}$ Institut für Virologie, Robert-Koch-Str. 17, D-35037 Marburg, Germany.

${ }^{3}$ Laboratory of Infectious Diseases, NIAID/NIH, 50 South Drive, Bethesda, Maryland 20892-8007, USA. ${ }^{4}$ Chiron Vaccines, Via Fiorentina 1, I-53100 Siena, Italy.

${ }^{5}$ Istituto di Microbiologia, Ospedale Luigi Sacco, Via Grassi 74, I-20175 Milano, Italy. ${ }^{6}$ These authors contributed equally to this work. Correspondence should be addressed to A.L. (lanzavecchia@irb.unisi.ch).

Published online 11 July 2004; doi:10.1038/nm1080 


\section{TECHNICAL REPORTS}

Table 1 Repertoire analysis of IgG memory B cells

\begin{tabular}{lcc}
$\begin{array}{l}\text { Months after } \\
\text { infection }\end{array}$ & \multicolumn{2}{c}{ Positive cultures/total cultures screened $(\%)^{\mathrm{a}}$} \\
2 & ELISA & Spike-staining \\
4 & $275 / 480(57.3 \%)$ & ND \\
6 & $123 / 480(25.6 \%)$ & $12 / 576(2.1 \%)$ \\
8 & $44 / 480(9.2 \%)$ & $21 / 768(2.7 \%)$ \\
\hline
\end{tabular}

aFraction of cultures screening positive in the SARS-CoV ELISA or staining SARS-CoV spike transfectants at different time points after infection. IgG ${ }^{+}$memory B cells were cultured at 10 cells per well in the presence of EBV and CpG 2006. Culture supernatants were analyzed after 2 weeks. There was no overlap between cultures screening positive by either assay, indicating that the assays detect distinct antibody specificities. SARS-CoV was not detectable in culture supernatant or in EBV-B cells, as determined by cytopathic assay on Vero cells and by RT-PCR (data not shown). ND, not determined because the assay was not available at the time of the analysis.

of SARS-CoV infection of Vero cells. Serum collected from the patient at different time points after infection scored positive in all three assays (see Supplementary Table 1 online), whereas sera from uninfected individuals were nonreactive. Antibodies detected by ELISA and staining of spike-transfectants were highest at 2 months after infection and decreased to about one-third by 8 months. In contrast, neutralizing antibodies remained constant with a titer of $1 / 128$. The isotype of the antibodies detected by ELISA and the spike-binding assay was IgG1 only.

Based on these data, we focused on $\mathrm{IgG}^{+}$memory B lymphocytes. These cells were isolated by a combination of magnetic and fluorescence-activated cell sorting and were immortalized with EBV in the presence of irradiated mononuclear cells $s^{6,21}$ and a CpG oligonucleotide (CpG 2006; ref. 22) that acts as a polyclonal activator of memory B cells ${ }^{23}$. We found that the addition of CpG 2006 increases the efficiency of B cell immortalization from 1-2\% to $30-100 \%$. Replicate cultures containing $10 \mathrm{IgG}^{+}$memory B cells were set up and culture supernatants were analyzed for the presence of specific antibodies after 2 weeks (Table 1). The fraction of cultures producing antibodies detected by ELISA was very high at 2 months after infection (virtually every second culture scored positive, consistent with a precursor frequency of $\sim 1$ in 20 ) and decreased by 6 and 8 months. The frequency of cultures producing antibodies staining spike-transfected cells could be measured only at the 4,6 and 8 months time points and was lower but more sustained. Most supernatants that stained spike-transfected cells showed neutralizing activity, but

Figure 1 Characterization of the SARS-CoV neutralizing S3.1 monoclonal antibody. (a) Staining of BHK cells transfected with SARS-CoV spike mRNA by purified S3.1 antibody (circles) and by the 6 months convalescent serum (squares). The filled symbols indicate the antibody concentration at which complete neutralization was observed. (b,c) Staining of SARS-CoV detected by immunoelectron microscopy. Top left, negative control; top right three panels, convalescent serum; bottom left, supernatant of a non-neutralizing B cell clone; bottom right three panels, S3.1 monoclonal antibody. Bar, $100 \mathrm{~nm}$. b

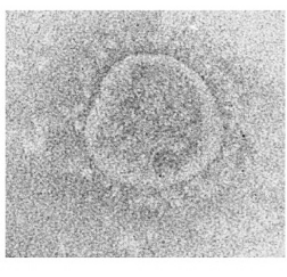

C

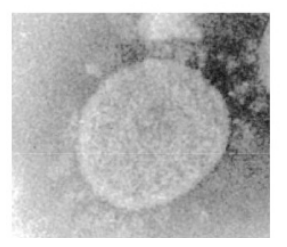

overall there was poor correlation between staining and neutralization (see Supplementary Fig. 1). These results indicate that only a small fraction of memory B cells specific for SARS-CoV antigens are directed against neutralizing epitopes present on the spike protein.

\section{Isolation of monoclonal antibodies to SARS-CoV}

EBV-B cells from cultures producing antibodies of the desired specificity were cloned by limiting dilution in the presence of irradiated mononuclear cells, with the addition of CpG 2006 to increase cloning efficiency. Out of 56 attempts, 43 (76\%) led to the isolation of one or more clones producing antibodies of the selected specificity (Table 2). The EBV clones were stable, and monoclonal antibodies were recovered in the culture supernatant at concentrations of 3-20 $\mu \mathrm{g} / \mathrm{ml}$.

Several of the clones that scored positive by ELISA produced antibodies specific for the SARS nucleoprotein (NP), whereas others did not recognize NP but stained SARS-CoV-infected cells. As expected, none of these antibodies showed neutralizing activity.

Initial attempts to isolate neutralizing monoclonal antibodies were limited by the use of a viral neutralization assay that requires a biosafety level 4 laboratory. Nonetheless, a culture showing neutralization titer was identified from the first screening and cloned, and one resulting clone, S3.1, produces an IgG1, $\mathrm{K}$ neutralizing monoclonal antibody. When purified from the culture supernatant and tested for its capacity to neutralize SARS-CoV, the S3.1 antibody neutralized $75 \mathrm{TCID}_{50}$ SARS-CoV at a concentration of $\sim 300 \mathrm{ng} / \mathrm{ml}$, a potency up to 300 -fold greater than that of convalescent serum (Fig. 1a). Furthermore, S3.1 neutralized with the same efficiency both the Frankfurt and Urbani isolates (data not shown) and decorated the spikes of SARS-CoV as detected by immunoelectron microscopy (Fig. 1b).

The later availability of SARS-CoV spike transfectants provided an efficient prescreening method. Approximately $50 \%$ of the antibodies staining spike-transfected cells also neutralized the homologous virus. a

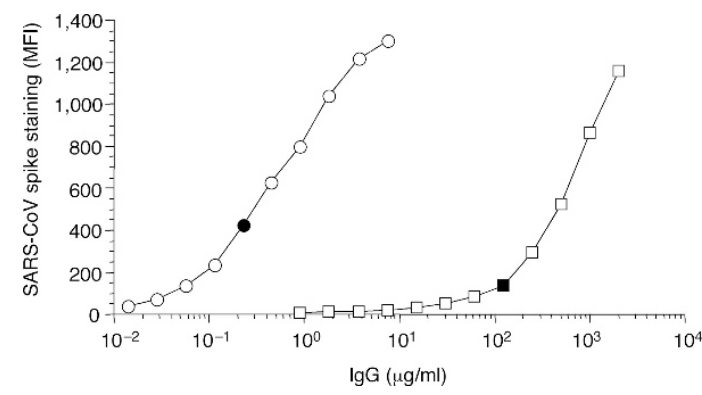

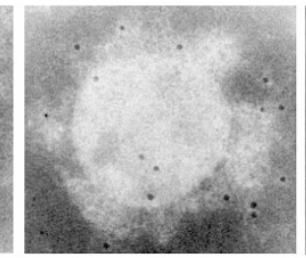
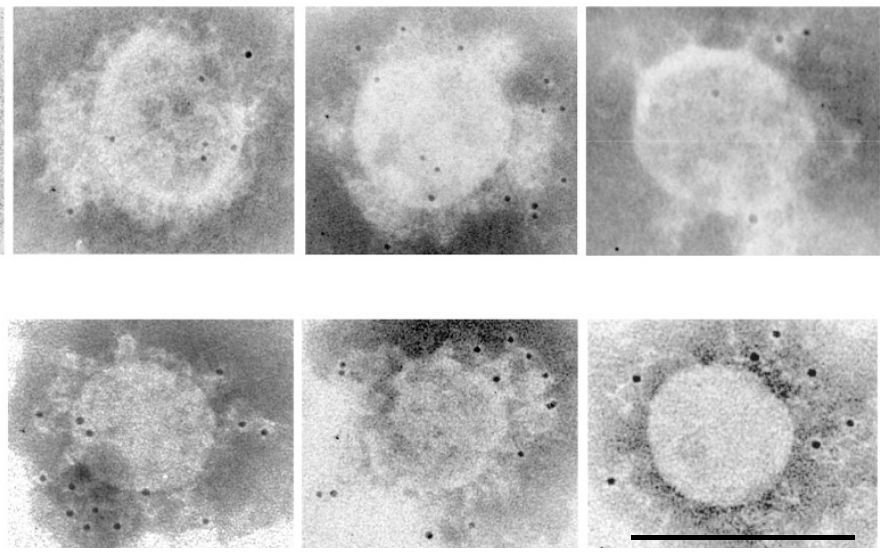
TECHNICAL REPORTS

We isolated 35 monoclonal antibodies that neutralized SARS-CoV (see examples in Table 2 and Supplementary Fig. 1). Some efficiently stained transfectants at low immunoglobulin concentrations, consistent with high-avidity binding, and showed a neutralizing titer proportional to the level of staining. In contrast, other antibodies stained poorly and only at high immunoglobulin concentrations, consistent with low-avidity binding, but showed efficient neutralization, even at immunoglobulin concentrations at which spike staining was hardly detectable. The final antibody concentration capable of completely neutralizing SARS-CoV infection in vitro ranged from 850 to $1 \mathrm{ng} / \mathrm{ml}$ (Table 2).

These results show that the method described for improved EBV immortalization can rapidly produce a battery of human monoclonal antibodies with potent neutralizing capacity in the subnanomolar range.

\section{S3.1 antibody inhibits SARS-CoV infection in mice}

The in vivo neutralizing activity of S3.1 antibody was tested in a mouse model of acute SARS-CoV infection ${ }^{24}$. Graded amounts of purified S3.1 monoclonal antibody were transferred to naive mice by intraperitoneal injection to determine whether antibody alone could prevent replication of SARS-CoV in the respiratory tract. A human IgG1 monoclonal antibody of irrelevant specificity (M12) was used as a negative control. After $2 \mathrm{~d}$, the mice received an intranasal challenge dose of $10^{4}$ TCID $_{50}$ of SARS-CoV (Table 3). Mice that received S3.1 monoclonal antibody were protected from replication of challenge virus, particularly in the lower respiratory tract. Significant $(P<0.05)$ restriction of virus replication in the upper respiratory tract was noted in those mice that received the highest dose of S3.1 monoclonal antibody. These results show that improved EBV transformation of memory B cells can lead to rapid and efficient isolation of candidate therapeutic monoclonal antibodies.

\section{DISCUSSION}

In this study we have demonstrated an improved method of B cell immortalization by EBV involving the addition of a polyclonal B cell activator, and we have shown that with this method it is possible to interrogate the memory $\mathrm{B}$ cell repertoire of a SARS convalescent patient and to isolate with high efficiency B cell clones producing neutralizing monoclonal antibodies. This procedure is rapid-it can be completed in less than 3 months - and allows screening of a large repertoire of antigen-specific memory $B$ cells for those with the most favorable affinity and specificity.

A high proportion of antigen-specific peripheral blood memory B cells, such as that found in the SARS convalescent patient, is not a prerequisite for the isolation of human monoclonal antibodies. Indeed, memory B lymphocytes are maintained through an individual's lifetime at levels that correlate with sustained serum antibody concentrations ${ }^{23}$. Consequently, the memory B cell pool can be interrogated at any time after priming, with the only limiting factor being the total number of cells that need to be screened to access enough specificities. As an example, we have been able to isolate neutralizing monoclonal antibodies specific for measles virus from a blood sample obtained 47 years after infection (E.T., unpublished data).

The high efficiency of EBV immortalization and cloning obtained in the presence of a polyclonal activator were instrumental in the isolation of several monoclonal antibodies specific for SARS-CoV. Most of the monoclonal antibodies we isolated using ELISA recognized NP or other viral proteins, but did not neutralize the virus. Indeed, the fraction of memory B cells that recognized denatured viral proteins exceeded by 5 - to 10 -fold the fraction of $\mathrm{B}$ cells that recognized the
Table 2 EBV immortalized B cell clones secreting monoclonal antibodies to SARS-CoV

\begin{tabular}{llcc}
\hline $\begin{array}{l}\text { Monoclonal } \\
\text { antibody }\end{array}$ & Isotype & Specificity & $\begin{array}{c}\text { Neutralizing } \\
\text { antibody concentration } \\
(n g\end{array}$ \\
\hline IgG/m|d
\end{tabular}

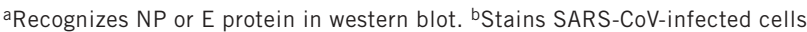
but specificity could not be determined. "Stains spike-transfected BHK cells. dIndicates final IgG concentration in culture conferring complete SARS-CoV neutralization. Data represent mean values of at least three determinations. n.n., non-neutralizing.

Table 3 Protection from virus replication in the mouse respiratory tract after transfer of the anti-SARS-CoV antibody S3.1

\begin{tabular}{lcccc}
\hline $\begin{array}{l}\text { Passive transfer } \\
\text { antibody }\end{array}$ & \multicolumn{4}{c}{ Virus replication in challenged mice } \\
\hline & \multicolumn{3}{c}{ Lungs } \\
& $\begin{array}{c}\text { No. infected/ } \\
\text { no. tested }\end{array}$ & $\begin{array}{c}\text { Mean ( } \pm \text { s.e.m.) } \\
\text { virus titer }\end{array}$ & $\begin{array}{c}\text { No. infected/ } \\
\text { no. tested }\end{array}$ & $\begin{array}{c}\text { Mean ( } \pm \text { s.e.m.) } \\
\text { virus titer }\end{array}$ \\
\hline S3.1 sup & $0 / 4$ & $\leq 1.5 \pm 0$ & $4 / 4$ & $4.8 \pm 0.32$ \\
S3.1, 800 $\mu \mathrm{g}$ & $0 / 4$ & $\leq 1.5 \pm 0$ & $2 / 4$ & $2.5 \pm 0.47$ \\
S3.1, 200 $\mu \mathrm{g}$ & $0 / 4$ & $\leq 1.5 \pm 0$ & $4 / 4$ & $3.4 \pm 0.41$ \\
S3.1, 50 $\mathrm{gg}$ & $2 / 4$ & $3.2 \pm 1.36$ & $4 / 4$ & $4.8 \pm 0.75$ \\
M12, 800 $\mu \mathrm{g}$ & $4 / 4$ & $7.5 \pm 0.1$ & $4 / 4$ & $6.4 \pm 0.41$ \\
\hline
\end{tabular}

The indicated amounts of purified antibody (in $500 \mu \mathrm{l}$ ) or \$3.1 culture supernatant (sup; $2 \mathrm{ml}, 10 \mu \mathrm{g} / \mathrm{ml}$ ) were administered to recipient mice by intraperitoneal injection $24 \mathrm{~h}$ before intranasal challenge with $10^{4} \mathrm{TCID}_{50}$ SARSCoV. Monoclonal antibody M12 (human IgG1, א) was injected as a negative control. Virus titers measured after $2 \mathrm{~d}$ are expressed as $\log _{10} \mathrm{TCID}_{50}$ per gram of tissue. The lower limit of detection of infectious virus in a $10 \% \mathrm{w} / \mathrm{v}$ suspension of lung homogenate was $1.5 \log _{10} \mathrm{TCID}_{50}$ per $\mathrm{g}$ and in $5 \% \mathrm{w} / \mathrm{v}$ suspension of nasal turbinates was $1.8 \log _{10} T_{C I D}$ per $g$. Comparisons that were statistically significantly different $(P<0.05)$ in a Student's $t$-test were: in lungs, $800 \mu \mathrm{g} \mathrm{S3.1}$ versus $800 \mu \mathrm{g} \mathrm{M12,} 200 \mu \mathrm{g} \mathrm{S} 3.1$ versus $800 \mu \mathrm{g} \mathrm{M12}$, and $50 \mu \mathrm{g}$ S 3.1 versus 800 $\mu \mathrm{g} \mathrm{M12}$; in nasal turbinates, $800 \mu \mathrm{g} \mathrm{S3.1}$ versus $800 \mu \mathrm{g} \mathrm{M} 12$ and $200 \mu \mathrm{g} \mathrm{S} 3.1$ versus $800 \mu \mathrm{g} M 12$. 


\section{TECHNICAL REPORTS}

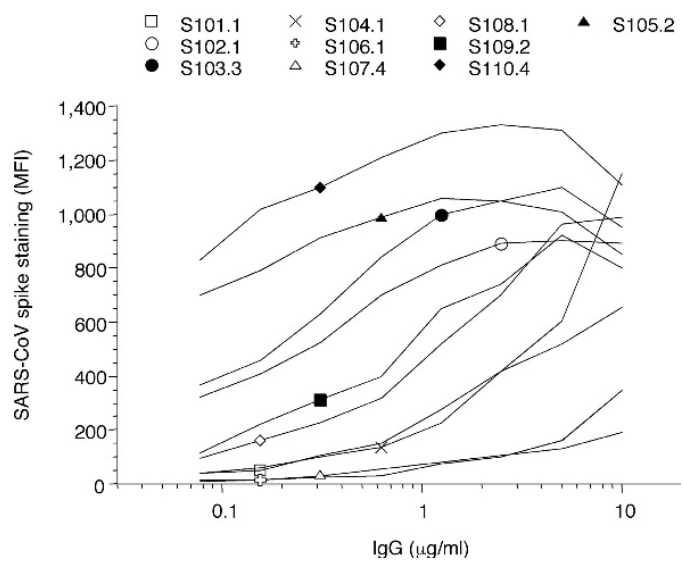

native spike protein. Furthermore, only $50 \%$ of the antibodies that stained spike-transfected cells showed neutralizing activity, possibly because they recognized epitopes that are not available on the spike trimers. These findings indicate that most of the human B cell response is directed against nonprotective epitopes.

Initial screening for neutralizing antibodies led to the isolation of the S3.1 clone, the only clone tested in an animal model. The subsequent development of a spike-binding assay allowed much more rapid identification of neutralizing candidates and led to the isolation of antibodies with neutralizing capacity up to 300 -fold higher. When neutralizing monoclonal antibodies were compared for spike staining and neutralizing activity, two patterns emerged (Fig. 2). Some antibodies showed high-avidity binding to spike transfectants and a neutralizing titer proportional to the degree of binding. In contrast, other antibodies showed low-avidity binding in spite of efficient viral neutralization. It is possible that the former recognize exposed epitopes within the receptor binding S1 domain of the spike protein, whereas the latter recognize epitopes within the $\mathrm{S} 2$ domain, which is exposed only during viral fusion and may be poorly accessible in transfected cells $^{25}$. An alternative possibility is that spike molecules may be differentially assembled in viral particles compared to BHK cells. This may explain the lack of correlation between staining and neutralization, as well as the finding that most antibodies neutralized virus at concentrations at which staining was hardly detectable. Further studies, including assessment of direct binding to viral particles, analysis of escape mutants ${ }^{25}$ and epitope mapping using synthetic peptides, will be required to elucidate how antibodies to the spike protein neutralize SARS-CoV infection.

The in vivo neutralizing activity of the S3.1 monoclonal antibody was tested in a mouse model of acute SARS infection ${ }^{24}$. Intraperitoneal injection of as little as $20 \mu \mathrm{g}$ of S3.1 antibody completely protected mice from viral replication in the lower respiratory tract, whereas restriction of virus replication was observed in the upper respiratory tract. The observation that viral replication was more effectively prevented in the lower respiratory tract than in the upper respiratory tract is consistent with findings in similar passive transfer experiments with influenza A viruses and respiratory syncytial virus ${ }^{26,27}$.

In conclusion, we have described a method for the rapid and efficient isolation of neutralizing antibodies to an emerging pathogen. This method offers two unique features: First, the antibodies are produced by immortalization of memory B lymphocytes, which are stable over a lifetime and can easily be isolated from peripheral blood. This bypasses the need for antigen boosting required for the generation of hybridomas. Second, the antibody is isolated from a primed natural
Figure 2 Binding and neutralization capacity of monoclonal antibodies specific for SARS-CoV spike. Shown is the staining of BHK cells transfected with SARS-CoV spike mRNA by serial dilutions of ten neutralizing monoclonal antibodies. The symbols indicate the neutralizing titer: that is, the antibody dilution added in the neutralization assay that gave complete viral neutralization.

host who has survived the natural infection, eliminating the need for immunization of experimental animals, which may show different susceptibility and, therefore, different immune responses. This method delivers large numbers of antibodies that can immediately be selected for the most favorable profile according to criteria such as affinity, epitope specificity and propensity to generate escape mutants.

\section{METHODS}

Patient and cell cultures. After informed consent was obtained, peripheral blood was collected from a 35-year-old patient who had recovered from infection by SARS-CoV. $\mathrm{IgG}^{+}$memory B cells were isolated by binding to CD22 microbeads (Miltenyi) followed by depletion of cells carrying IgM, IgD and IgA by cell sorting. Memory B cells were seeded at 10 or 50 cells per well in $96 \mathrm{U}$ -

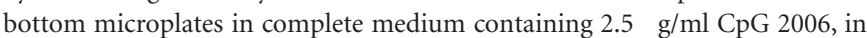
the presence of EBV (30\% supernatant of B95-8 cells) and irradiated allogeneic mononuclear cells (50,000 per well). After 2 weeks, the culture supernatants were screened for specific antibodies. Positive cultures were cloned by limiting dilution in the presence of CpG 2006 and irradiated mononuclear cells. Antibody was purified from culture supernatants by affinity chromatography on protein A columns (Amersham).

ELISA. The Frankfurt isolate of the SARS-CoV (GenBank accession number AY310120) was provided by H.-W. Doerr (University of Frankfurt). Cell culture supernatant of infected Vero cells was collected, clarified by centrifugation at 3,000 r.p.m. for $5 \mathrm{~min}$ and then centrifuged through a $20 \%$ sucrose cushion at 20,000 r.p.m. for $2 \mathrm{~h}$ in a Beckman SW28 rotor. The pellet was purified using a potassium tartrate/glycerol gradient and resuspended in $500 \mu \mathrm{l} \mathrm{TNE}$ buffer (10 $\mathrm{mM}$ Tris-HCl, $\mathrm{pH} 7.4,0.15 \mathrm{M} \mathrm{NaCl}, 2 \mathrm{mM}$ EDTA) to a protein concentration of approximately $0.5 \mathrm{mg}$ per $\mathrm{ml}^{28}$. The antigen suspension used for ELISA was prepared by adding $1 \%$ SDS to the viral pellet and boiling the mixture for 10 min. ELISA plates (Nunc) were coated with SARS-CoV antigen in $0.1 \mathrm{M}$ sodium phosphate buffer ( $\mathrm{pH}$ 7). Serial dilutions of serum or antibody supernatants were added for $2 \mathrm{~h}$ and the antibody bound was revealed using isotypespecific secondary antibodies coupled to alkaline phosphatase.

In vitro neutralization assay. Sera or culture supernatants were diluted in 2-log steps and $25 \mu \mathrm{l}$ was mixed with an equal volume of medium containing 75 $\mathrm{TCID}_{50}$ SARS-CoV (virus titer was determined according to the method of Karber $\left.{ }^{29}\right)$. Mixtures were allowed to sit $45 \mathrm{~min}$ at room temperature, and then $50 \mu$ of Vero cells $\left(1.5 \times 10^{5}\right.$ per $\left.\mathrm{ml}\right)$ were added and the cultures incubated for $3 \mathrm{~d}$ at $37^{\circ} \mathrm{C}$. All assays were performed in a biosafety level 4 laboratory. As neutralization titer we indicate the last antibody dilution that completely prevents the cytopathic effect of SARS-CoV. As neutralizing antibody concentration we indicate the final antibody concentration $(\mathrm{ng} / \mathrm{ml})$ in the cultures at which complete protection was observed.

Transient expression of the spike protein of SARS-CoV. cDNA was prepared from total SARS-CoV RNA using random primers. The coding region of the spike protein was amplified by PCR. The forward and reverse PCR primers overlap 20 nucleotides of $5^{\prime}$ and $3^{\prime}$ termini, respectively, of the spike protein coding region (GenBank accession number AY310120). To ensure translation in eukaryotic cells, a canonical Kozak sequence (5'-GCCGCCACC-3') was included in front of the protein's start codon. The amplified fragment was inserted into an alphavirus vector as described ${ }^{30}$. The recombinant plasmid VCR-SSP was transcribed in vitro using the MMESSAGE MMACHINE kit (Ambion). The RNA obtained from $2 \mu \mathrm{g}$ of the plasmid DNA in a $20-\mu \mathrm{l}$ reaction was mixed with 20 million BHK cells in $1 \mathrm{ml}$ of PBS. Cells were electroporated using GenePulser (Bio-Rad) twice under the condition of 1,650 V/25 


\section{TECHNICAL REPORTS}

microfarads. Cells were cultured in DMEM 10\% FCS overnight and stained with serial dilutions of serum or culture supernatant followed by Cy5-labeled anti-human IgG antibodies (Jackson Laboratories).

Immunoelectron microscopy. Clarified supernatant (200 $\mu \mathrm{l})$ of SARS-CoVinfected Vero cells was mixed with $200 \mu \mathrm{l}$ of culture supernatant or serum for 30 min at room temperature. A drop of the suspension was deposited on formvar carbon-coated nickel grids for $1 \mathrm{~min}$. The excess fluid was blotted away and the grids were floated on a drop of PBS containing $1 \%$ BSA for $10 \mathrm{~min}$. Bound antibodies were detected with a donkey anti-human IgG antibody coupled to 6-nm gold particles (Dianova). The samples were negatively stained with $2 \%$ phosphotungstic acid and examined in a Zeiss 109 electron microscope.

In vivo neutralization assay. The SARS-CoV (Urbani strain) was kindly provided by L.J. Anderson and T.G. Ksiazek (Centers for Disease Control and Prevention, Atlanta, Georgia). A virus stock of $10^{6.5} \mathrm{TCID}_{50}$ per $\mathrm{ml}$ was generated. All work with infectious virus was performed inside a biosafety cabinet in a biosafety containment level 3 facility and personnel wore powered air purifying respirators (3M HEPA AirMate). The mouse studies were approved by the NIH Animal Care and Use Committee and were carried out in an approved animal biosafety level 3 facility. All personnel entering the facility wore powered air purifying respirators. Four- to six-week-old female BALB/c mice (Taconic) were housed four mice per cage. On day 0 , mice were lightly anesthetized with isoflurane and received intraperitoneal injections of the S3.1 antibody in one of three doses $(800,200,50 \mu \mathrm{g})$ or a control monoclonal antibody lacking neutralizing activity. Mice were challenged with $10^{4} \mathrm{TCID}_{50}$ of SARS-CoV intranasally $24 \mathrm{~h}$ later, and were killed after an additional $2 \mathrm{~d}$. The lungs and turbinates were removed and homogenized in a 5\% w/v suspension in Leibovitz 15 medium (Invitrogen) and virus titer was determined in Vero cell monolayers.

Note: Supplementary information is available on the Nature Medicine website.

\section{ACKNOWLEDGMENTS}

We thank J. McAuliffe and L. Vogel from the Laboratory of Infectious Diseases, NIAID, for expert assistance in performing the animal studies. We thank I. Giacchetto for expert technical assistance and F. Sallusto for critical reading and comments. A.L. is supported by the Helmut Horten Foundation.

\section{COMPETING INTERESTS STATEMENT}

The authors declare competing financial interests (see the Nature Medicine web site for details).

Received 9 November 2003; accepted 26 March 2004

Published online at http://www.nature.com/naturemedicine/

1. Behring, E. \& Kitasato, S. [On the development of immunity to diphtheria and tetanus in animals]. Dtsch Med. Wochenschr. 90, 2183 (1965).

2. Keller, M.A. \& Stiehm, E.R. Passive immunity in prevention and treatment of infectious diseases. Clin. Microbiol. Rev. 13, 602-614 (2000).

3. Kohler, G. \& Milstein, C. Continuous cultures of fused cells secreting antibody of predefined specificity. Nature 256, 495-497 (1975).

4. Steinitz, M., Klein, G., Koskimies, S. \& Makel, O. EB virus-induced B lymphocyte cell lines producing specific antibody. Nature 269, 420-422 (1977).

5. Kozbor, D. \& Roder, J.C. Requirements for the establishment of high-titered human monoclonal antibodies against tetanus toxoid using the Epstein-Barr virus technique. J. Immunol. 127, 1275-1280 (1981).

6. Lanzavecchia, A. Antigen-specific interaction between T and B cells. Nature $\mathbf{3 1 4}$ 537-539 (1985).

7. Kozbor, D., Roder, J.C., Chang, T.H., Steplewski, Z. \& Koprowski, H. Human antitetanus toxoid monoclonal antibody secreted by EBV-transformed human B cells fused with murine myeloma. Hybridoma 1, 323-328 (1982).

8. Karpas, A., Dremucheva, A. \& Czepulkowski, B.H. A human myeloma cell line suitable for the generation of human monoclonal antibodies. Proc. Natl. Acad. Sci. USA 98, 1799-1804 (2001).

9. Jones, P.T., Dear, P.H., Foote, J., Neuberger, M.S. \& Winter, G. Replacing the complementarity-determining regions in a human antibody with those from a mouse. Nature 321, 522-525 (1986).

10. McCafferty, J., Griffiths, A.D., Winter, G. \& Chiswell, D.J. Phage antibodies: filamentous phage displaying antibody variable domains. Nature 348, 552-554 (1990).

11. Green, L.L. Antibody engineering via genetic engineering of the mouse: XenoMouse strains are a vehicle for the facile generation of therapeutic human monoclonal antibodies. J. Immunol. Methods 231, 11-23 (1999).

12. Brekke, O.H. \& Sandlie, I. Therapeutic antibodies for human diseases at the dawn of the twenty-first century. Nat. Rev. Drug Discov. 2, 52-62 (2003).

13. Johnson, S. et al. Development of a humanized monoclonal antibody (MEDI-493) with potent in vitro and in vivo activity against respiratory syncytial virus. J. Infect. Dis. 176, 1215-1224 (1997).

14. Drosten, C. et al. Identification of a novel coronavirus in patients with severe acute respiratory syndrome. N. Engl. J. Med. 348, 1967-1976 (2003).

15. Ksiazek, T.G. et al. A novel coronavirus associated with severe acute respiratory syndrome. N. Engl. J. Med. 348, 1953-1966 (2003).

16. Rota, P.A. et al. Characterization of a novel coronavirus associated with severe acute respiratory syndrome. Science 300, 1394-1399 (2003).

17. Fouchier, R.A. et al. Aetiology: Koch's postulates fulfilled for SARS virus. Nature 423, 240 (2003).

18. Poutanen, S.M. et al. Identification of severe acute respiratory syndrome in Canada. N. Engl. J. Med. 348, 1995-2005 (2003).

19. Lee, N. et al. A major outbreak of severe acute respiratory syndrome in Hong Kong. N. Engl. J. Med. 348, 1986-1994 (2003).

20. Donnelly, C.A. et al. Epidemiological determinants of spread of causal agent of severe acute respiratory syndrome in Hong Kong. Lancet 361, 1761-1766 (2003).

21. Giachino, C., Padovan, E. \& Lanzavecchia, A. $\kappa^{+} \lambda^{+}$dual receptor $B$ cells are present in the human peripheral repertoire. J. Exp. Med. 181, 1245-1250 (1995).

22. Hartmann, G. \& Krieg, A.M. Mechanism and function of a newly identified CpG DNA motif in human primary B cells. J. Immunol. 164, 944-953 (2000).

23. Bernasconi, N.L., Traggiai, E. \& Lanzavecchia, A. Maintenance of serological memory by polyclonal activation of human memory B cells. Science 298, 2199-2202 (2002).

24. Subbarao, K. et al. Prior infection and passive transfer of neutralizing antibody protect mice from subsequent infection with SARS coronavirus. J. Virol. 78, 3572-3577 (2004)

25. Gebauer, F. et al. Residues involved in the antigenic sites of transmissible gastroenteritis coronavirus S glycoprotein. Virology 183, 225-238 (1991).

26. Graham, B.S., Perkins, M.D., Wright, P.F. \& Karzon, D.T. Primary respiratory syncytial virus infection in mice. J. Med. Virol. 26, 153-162 (1988).

27. Stack, A.M. et al. Primary respiratory syncytial virus infection: pathology, immune response, and evaluation of vaccine challenge strains in a new mouse model. Vaccine 18, 1412-1418 (2000)

28. Becker, S., Feldmann, H., Will, C. \& Slenczka, W. Evidence for occurrence of filovirus antibodies in humans and imported monkeys: do subclinical filovirus infections occur worldwide? Med. Microbiol. Immunol. (Berl.) 181, 43-55 (1992).

29. Karber, G. 50\% end-point calculation. Arch. Exp. Pathol. Pharmak. 162, 480-483 (1931).

30. Perri, S. et al. An alphavirus replicon particle chimera derived from Venezuelan equine encephalitis and Sindbis viruses is a potent gene-based vaccine delivery vector. J. Virol. 77, 10394-10403 (2003). 